\title{
Reflexiones sobre la formación docente de una profesora-administradora
}

\author{
Ruth Torres Carrasco
}

\section{Resumen}

Este texto tiene como objetivo presentar una aproximación conceptual a la formación docente, con la intención de motivar al lector -educador, profesor y aquellos que no han tenido algún acercamiento o no son expertos en este tema- a reflexionar acerca de la importancia que tiene este proceso en el desempeño de la función educativa, particularmente en el nivel universitario. Para ello es necesaria una breve distinción de los conceptos educación y formación, y de cómo el primero puede contribuir o ser el punto de partida para enmarcar e identificar el trabajo del segundo. Aquí también se presentan algunas concepciones que diversos autores hacen de la formación docente usando el término como un sinónimo de educación, y otros más que la utilizan ligada a un campo específico para su desempeño.

Palabras clave: formación docente, educación, profesora.

\section{REFLECTIONS ON TEACHER TRAINING FROM A TEACHER-ADMINISTRATOR}

\begin{abstract}
This text presents a conceptual approach to teacher training. The purpose is to motivate readers, teachers, and people with no experience on this topic to think about the importance of teacher training and its impact on the performance of educational actions, particularly at the university level. The differences between education and teacher training are discussed, and how the former can frame the meaning of the later. Several teacher-training definitions are presented.
\end{abstract}

Key words: epigenetics, pollution, transgenerational, inheritance, genetics. 


\section{Ruth Torres Carrasco}

rtorresc@unam.mx

Doctora en Educación por la Facultad de Educación de la Universidad Complutense de Madrid, España. Licenciada y Maestra en Administración, por la Facultad de Contaduría y Administración (FCA) de la Universidad Nacional Autónoma de México. Ha realizado actividades de gestión institucional y desarrollo académico entre las que se destacan el rediseño curricular, creación de programas académicos para la formación de profesores y estudiantes, evaluación de programas institucionales, gestión académico-administrativa, entre otras. Es profesora de tiempo completo definitiva por oposición en el área de Recursos Humanos en la FCA-UNAM.

\section{Educación y formación}

Resulta complejo distinguir algunos conceptos que en lo cotidiano suelen identificarse como sinónimos. Educación y formación son ejemplo de ello, en particular en el campo educativo en el cual no son sinónimos, aunque en la práctica o en su uso habitual se presenten como tales. Para aclarar un poco estos conceptos, diremos, como muestra, que es común oír que se inscribe a un niño a determinada escuela para que "tenga una buena formación", aunque podría decirse también "para que tenga una buena educación", lo que nos hace pensar que el significado es el mismo.

Otros casos muestran que el uso indistinto de estas acepciones ya no es tan sencillo; así, algunas universidades imparten la carrera de Ciencias de la Educación y nos parecería extraño, por decir lo menos, oír que alguien estudia Ciencias de la Formación.

En algunos campos del saber se utiliza la palabra formación para referirse al modo de acceso al conocimiento de éstos; por ejemplo, en la formación psicoanalítica o la formación docente. En los casos anteriores sería raro decir educación docente o enseñanza docente; pero estos ejemplos nos dan la idea de que se trata de procesos diferentes y que, por ello, es necesario delimitar a la formación como un proceso de preparación para el ejercicio docente, por un lado; y para el ejercicio de determinada profesión, como el psicoanálisis, por el otro. 
Etimológicamente, la palabra educación (del sustantivo educatio, educationis) procede del latín educare, que significa "criar, nutrir o alimentar, dirigir, encaminar, dictrinar", y de exducere, que equivale a "sacar, llevar o conducir desde adentro hacia fuera" (Rosales Medrano \& Rosales Badilla, 2013). De acuerdo con Rodríguez (Rodríguez López, 2000) esta doble etimología ha provocado el nacimiento de dos acepciones que, en principio, aparecen como opuestas pero en realidad comparten tanto el concepto de educación como el de formación. En la primera definición, la educación es un proceso de incremento, de proximidad desde fuera; en la segunda, se trata de una conducción, de un encausamiento de las disposiciones ya existentes en el sujeto para su uso hacia afuera.

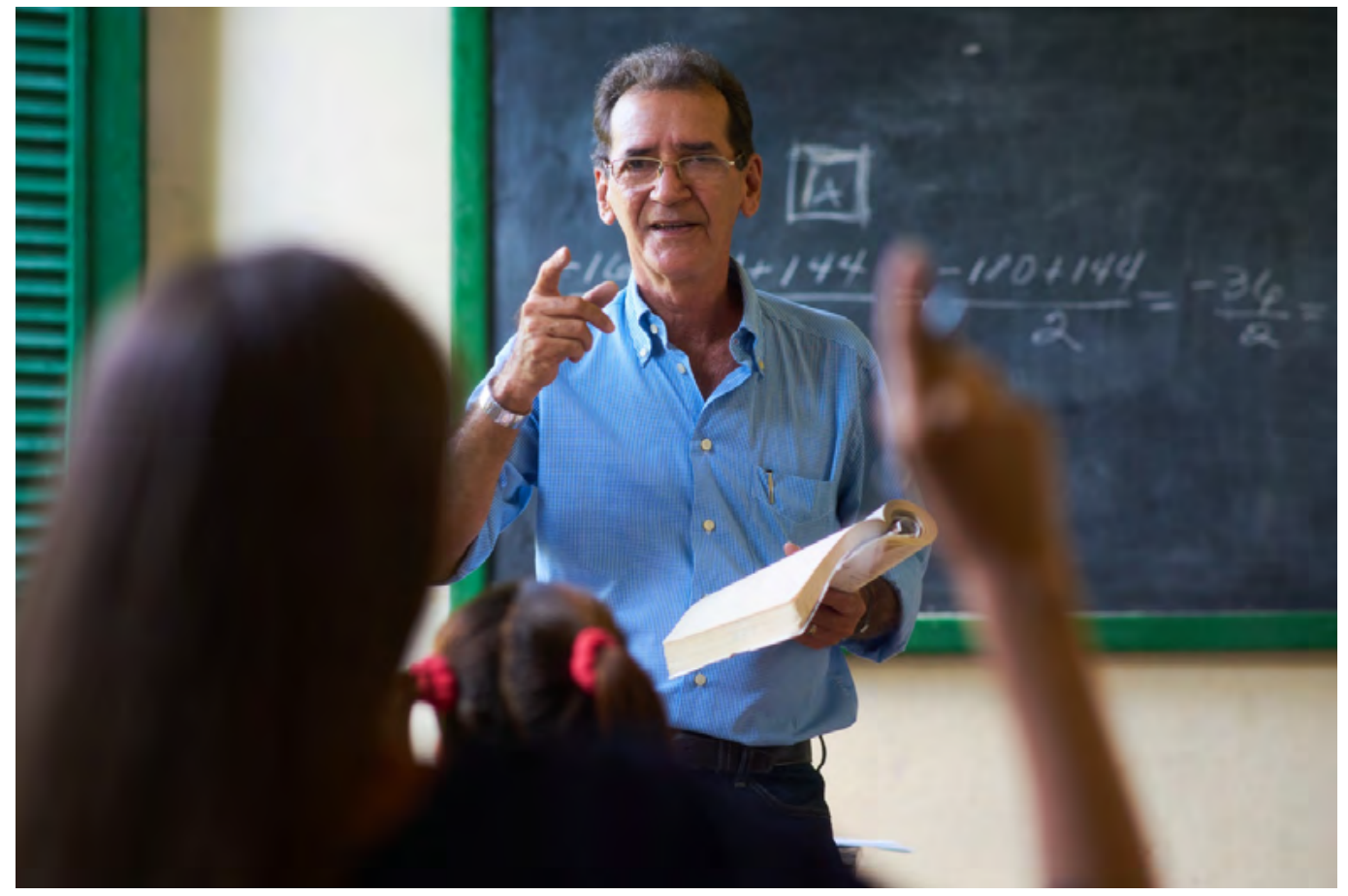

Por otro lado, la palabra formación (del sustantivo formatio, formationis) proviene de la raíz latina formare, que significa "hacer algo dándole una forma que le es propia"; es un proceso que provoca la aparición de algo que no existía; cuando una persona adquiere un desarrollo, una actitud o una habilidad obtiene una formación que incluso pude preparar a otro

De acuerdo con Aragón (Aragón Melchor, Méndez Barriga, \& Morales Reyes, 2013) el concepto de formación se emplea para referirse a la educación, preparación,o enseñanza en un determinado quehacer. En los países anglosajones en los que se inició el uso de este término (para después extenderse de manera general a otras latitudes) significa educación o entrenamiento, por lo que se puede entender como una función social de transmisión de conocimientos, habilidades y competencias suficientes para que las personas se integren a las actividades de producción de bienes o servicios e incluso a actividades relacionadas con aspectos culturales. Esta concepción es la que, en las disciplinas ad- 
ministrativas, tiene un mayor uso, pues es el fundamento con el que se conciben los procesos de capacitación.

Para Marcelo García (1989), "la formación puede entenderse como un proceso de desarrollo y de estructuración de la persona que se lleva a cabo bajo el doble efecto de la maduración interna y las posibilidades de aprendizaje y de experiencias de los sujetos", por lo que el concepto formación tiene un sentido amplio que se refiere al proceso de conducción, adiestramiento e instrucción orientado a la valorización técnica, humana y cultural; así mismo, este autor afirma que tiene que ver con el conjunto de actividades indispensables, previamente seleccionadas, mediante las cuales se transmiten al individuo conocimientos, habilidades y actitudes necesarias para desempeñar una función o profesión de interés social, empresarial e institucional.

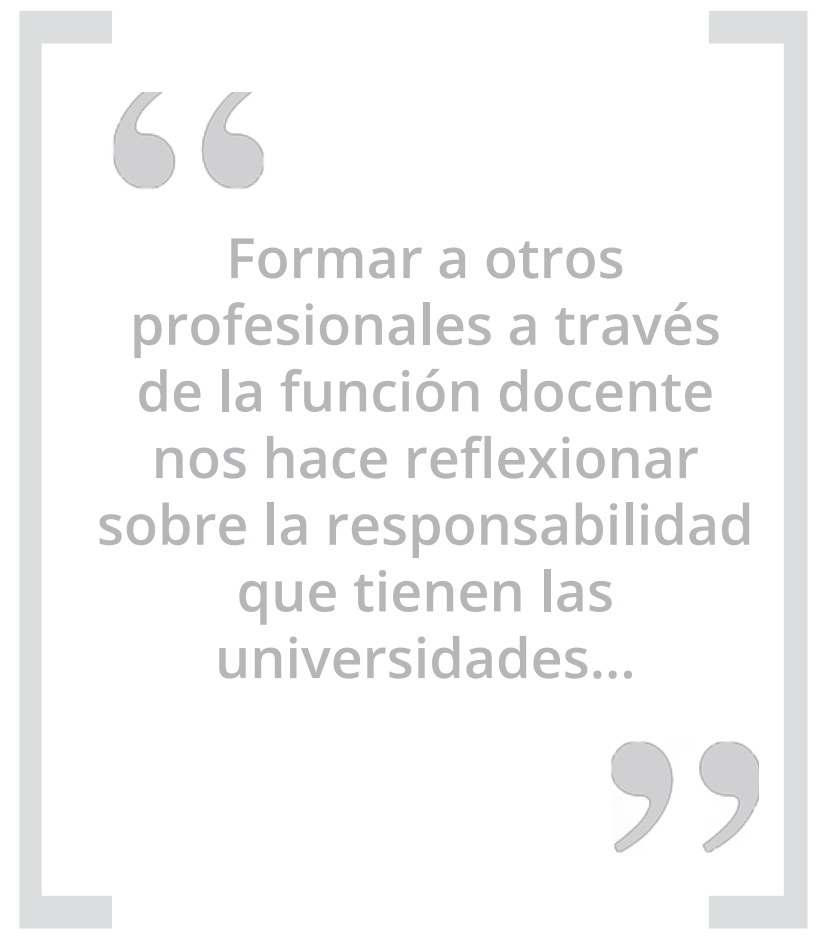

Desde la perspectiva de los modelos pedagógicos, se ha considerado a la formación como el proceso permanente de adquisición, estructuración y reestructuración de conductas -conocimientos, habilidades, valores- (Aragón Melchor, Méndez Barriga, \& Morales Reyes, 2013) para el desempeño de la docencia, por lo que damos cuenta de que formación docente se vincula con la función docente que tienen como propósito la enseñanza de determinada disciplina y para formar a otros en ella.

Hay que destacar que la formación del profesorado debe estar inmersa en un proceso continuo circunscrito en el campo de la educación y de las ciencias de la educación (Ferreres \& Imbernón, sin fecha); incluso dentro de la formación permanente existe una serie de términos muy utilizados como sinónimos que hacen referencia a esta actividad tales como: educación permanente, formación permanente, formación continua, formación en ejercicio, formación técnico-profesional, entre otros.

De lo anterior, se desprende que se considera a la formación permanente de los profesores como un subsistema específico de formación en una institución educativa que se dirige al perfeccionamiento de la tarea educativa con la finalidad de conseguir un mejoramiento de ella y que le permita adecuarse a los cambios científicos, disciplinares y sociales de su entorno. La formación pretende, en un inicio, que los profesores se cuestionen a partir de su propia práctica, los procesos que sustentan la función docente como eje fundamental de su propio desempeño.

Si se parte de los conceptos anteriores -educación y formación-, el ejer- 
cicio de la función docente universitaria requiere de las dos concepciones, la de educación en un campo disciplinario y la de formación didáctico-pedagógica para su transmisión, ya que ambas instruirán (nutrirán) al docente, ofreciéndole un desarrollo de facultades cognitivas, intelectuales y técnicas que posteriormente utilizará para el perfeccionamiento de una actividad en la cual deba ser apto o adquiera la habilidad para llevarla a cabo. La docencia adopta entonces diversas características educativas y formativas que se manifiestan en el ejercicio de esta actividad cuya observación muestra que la práctica docente es heterogénea y que no se ajusta a un modelo uniforme de trabajo ya que la enseñanza varía de una disciplina a otra.

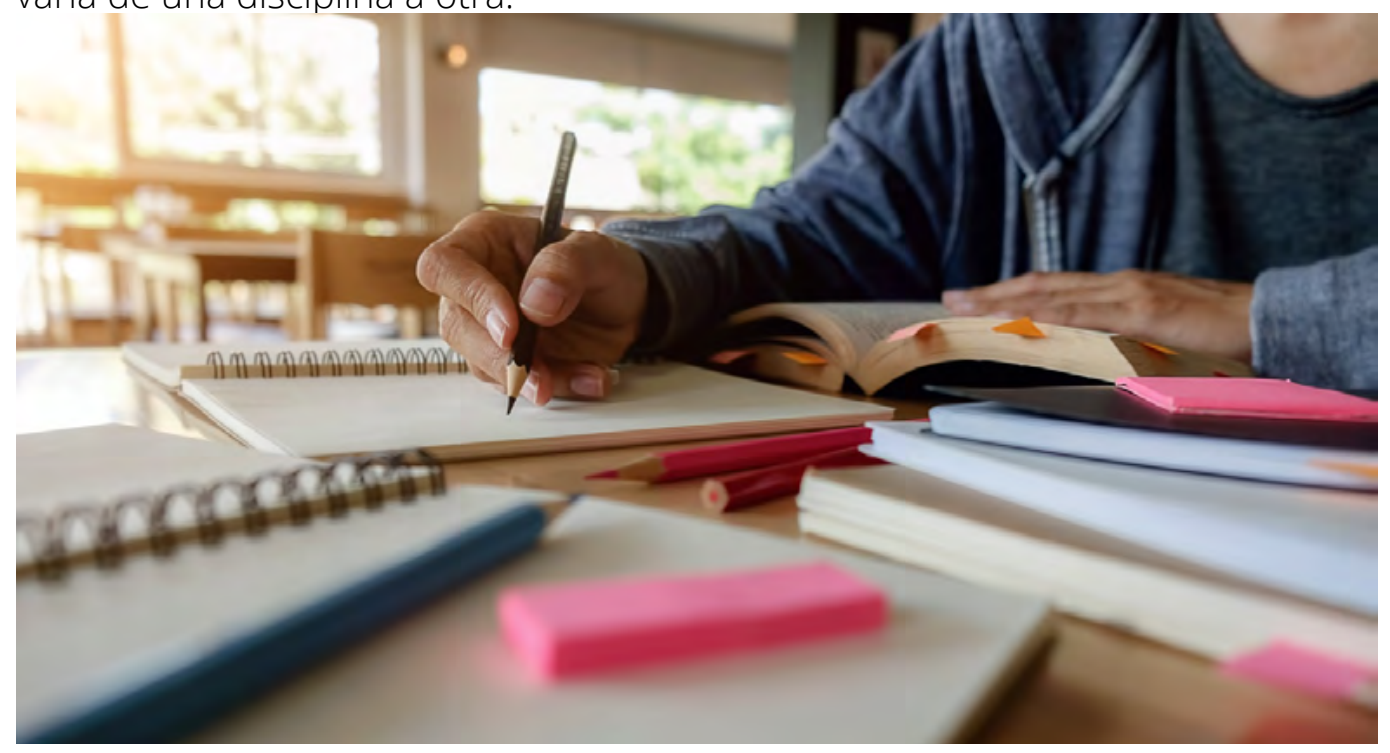

\section{La función docente universitaria su vínculo con la formación para su ejercicio}

La función docente es una de las actividades sociales más importantes en el desarrollo de la humanidad. La docencia es considerada por varios autores y estudiosos del tema como difícil, compleja, ardua, intrincada e incluso poco valorada desde el punto de vista económico. En el caso de la docencia universitaria, y los profesores responsables de ella, se desarrollan otras actividades que hacen todavía más difícil el ejercicio de su función, ya que antes de ser docentes

1. Lo que representa, en muchos casos, su actividad

principal en el mercado laboral y de la cual obtienen sus mayores ingresos económicos. son profesionales ${ }^{1}$ en alguna rama del conocimiento misma que transmiten a jóvenes que se encuentran en una etapa formativa para la adquisición de esos conocimientos científicos y técnicos que les permitirán ejercer una profesión.

Formar a otros profesionales a través de la función docente nos hace reflexionar sobre la responsabilidad que tienen las universidades y quienes en ellas enseñan; los docentes y su ejercicio desempeñan un doble papel: transmitir los conocimientos técnicos o científicos para resolver los problemas y para 
Ésta es una pregunta que puede tener varias respuestas que pueden ser el resultado de procesos de reflexión colectiva entre profesores que estén inmersos en tareas de formación docente. Una de las respuestas implica hacer una autoanálisis de la trayectoria y el desempeño en la tarea de enseñar, cómo se llegó a ella y qué motivaciones o razones propician el compromiso de permanecer ejerciéndola. La función docente se destina a un grupo social en el cual conviven diferentes tipos de formaciones, personalidades, intereses, apreciaciones sobre el tema, grupos humanos, complejidad de las disciplinas, conocimiento del proyecto educativo de la institución donde se realizará, entre otros aspectos; lo que hace complicada la dinámica de "enseñar a enseñar". Es aquí donde el profesor universitario requiere de una preparación que le provea de metodologías, técnicas o herramientas didáctico-pedagógicas que le auxilien en su misión de enseñar.

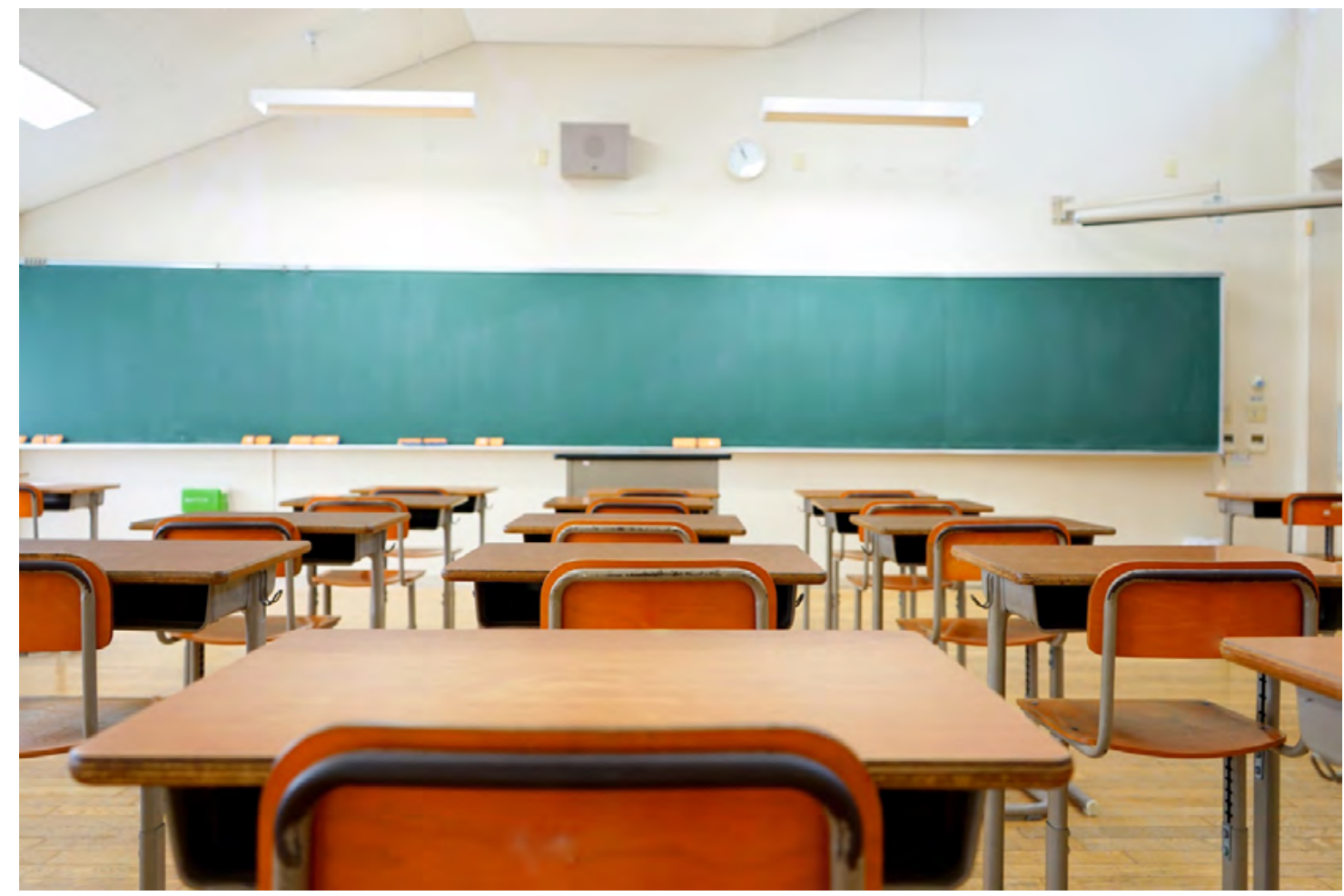

Si bien es cierto que para ejercer la educación superior se requiere del conocimiento y la formación académica-profesional en ciertas disciplinas, también es cierto que la mayoría de los egresados de las licenciaturas en nuestro país que se incorporan al trabajo docente no poseen una preparación mínima básica para ejercer la docencia, debido a que, en general, los currículos de las carreras no incluyen asignaturas que les permitan adquirir los conocimientos y las habilidades para el desempeño de esta tarea (salvo aquellas profesiones como la psicología educativa o pedagogía), por lo tanto, esta actividad se hace un tanto improvisada, poco reflexiva y propicia que se repitan modelos que pueden obstaculizar la enseñanza o el aprendizaje; de lo anterior, se des- 
prende que es indispensable que quien se dedica a la función docente en la educación superior se instruya didáctica y metodológicamente en la tarea de enseñar, por lo mismo es necesario invertir tiempo y esfuerzo para formarse como docentes.

La formación docente debe orientarse a ofrecer a los profesores los conocimientos teóricos-metodológicos y habilidades necesarios para aplicar en sus clases, las directrices establecidas en las normativas oficiales, así como propiciar un desarrollo profesional de los profesores que les facilite autonomía y la capacidad para tomar decisiones en cuanto a la construcción y elaboración de los conocimientos establecidos en el currículum. La formación docente no debe concebirse como algo puntual sino como un proceso de cambio y éste como un proceso de crecimiento personal (Rodríguez López, 2000) para el ejercicio de una docencia reflexiva y emancipadora.

\section{Conclusiones}

La formación implica algo intelectual y razonado, al mismo tiempo requiere de una serie de tareas que van desde una preparación disciplinar fundamental hasta el dominio de los tecnicismos propios de una profesión, pasando por el dominio de las técnicas del saber enseñar. Así pues, el primer problema que se ha de analizar en cualquier institución de educación superior y en particular en las universidades, es la formación del profesorado que estará en sus aulas, talleres y laboratorios. Existe una polémica respecto a lo que se denomina formación en los "programas de formación de profesores", que se diferencian del entrenamiento y la capacitación, lo cual hace necesario analizar si la concepción pedagógica que los orienta, tiene una visión normativa y prescriptiva del conjunto de conocimientos que reclama el saber docente.

Una interrogante de interés en este sentido nos dice lo siguiente: ¿cuál es la formación de quienes se presentan como docentes del profesor universitario? Esto es, qué tipo de saber, qué tipo de experiencia y qué tipo de cultura se requiere para ser profesor de docentes universitarios, que a su vez formarán profesionistas que ejercerán un trabajo útil para la sociedad. La formación de profesores, por ende, es una actividad preponderante en toda institución de educación superior, por lo que es deseable que desde las estructuras organizacionales de las universidades, se facilite y se creen los espacios para que faciliten la inserción de los docentes a esta vital tarea universitaria.

El docente que ejerce esta función tiene un conocimiento disciplinar, aunque le falte desarrollar el aspecto formativo como profesor para ver a la actividad docente como otra profesión y no como un complemento a cualquier otra de carácter profesionalizante como lo fue en sus inicios el ejercicio docente en la universidad; éste tiene que ser uno de los objetivos de los que se compongan 
los programas de formación del profesorado. Esto llevará a los profesores a realizar una tarea educativa más situada y acorde con la disciplina.

En esta misma línea en la que se ve a la docencia como una profesión, con todas las incidencias que comprende, varios autores estudian la práctica docente como una profesión. Lo que ahora se llama profesionalización de la docencia se inicia con el análisis de la función docente como punto de partida, se transita por los aspectos técnico-instrumentales de la formación, se continua con la actualización docente y se debe llegar a la profesionalización, proceso de enorme importancia para el ejercicio de esta actividad en el nivel universitario.

En resumen, la formación docente es un proceso social de reflexión sobre la educación y el para qué, donde el profesor debe poseer conocimientos sobre una ciencia, una disciplina o un arte (formación académica) que combinados con aspectos epistemológicos, pedagógicos y didácticos, le permitan desarrollar las habilidades y capacidades necesarias en la transmisión de conocimientos, para la formación de futuros profesionales y para encaminarse a la profesionalización de la docencia universitaria.

\section{Bibliografía}

* Aragón Melchor, J., Méndez Barriga, V., \& Morales Reyes, R. (2013). Producción - Formación del Docente ¿Olvido en las tendencias pedagógicas? En Rosales (Coord.), Teoría y práctica de la profesión docente. Universidad Pedagógica del Estado de Sinaloa (pp. 37-55).

* Chehaybar y Kuri, E. (2003). Hacia el futuro de la formación docente en educación superior. México: Plaza y Valdés Editores.

* Díaz Barriga, Á. (1996). Investigación educativa y formación de profesores. Contradicciones de una articulación. Cuadernos del CESU. México: Universidad Nacional Autónoma de México.

Eusse Zuluaga, O. (1993). Memorias. CISE-UNAM.

* Fernández Pérez, J. (2013). Formación y práctica docente. Un estudio sobre egresados de posgrado. (B. U. Tlaxcala., Ed.) México: Ediciones Días de Santos.

* Ferreres, V., \& Imbernón, F. (sin fecha). Formación y actualización para la función pedagógica. (D. y. escolar, Ed.) Madrid, Síntesis Educación.

- Ferry, G. (2000). El trayecto de la formación. Los enseñantes entre la teoría y la práctica. México: Paidós-Educador.

* Furlán, A. (1987). Conceptos y problemas de la formación de profesores (algunos puntos críticos). Material de trabajo del Foro Nacional sobre Formación de Profesores. (UNAM, Recopilador) México. 
* García, C. M. (1989). Introducción a la formación del profesorado. Teoría y métodos. Sevilla: Universidad de Sevilla.

- Grediaga Kuri, R. (2000). Profesión Académica, disciplinas y organizaciones. (ANUIES, Ed.) México: Asociación Nacional de Universidades e Instituciones de Educación Superior.

* Honoré, B. (1980). Para una teoría de la formación. Madrid: Narcea De Ediciones.

- Latapí Sarre, P. (2009). Finale prestissimo. Pensamientos, vivencias y testimonios. México: Fondo de Cultura Económica.

* Morán Oviedo, P. (1999). La docencia como actividad profesional. México: Ediciones Gernika.

* Moreno Olivos, T. (2012). Si los buenos profesores "no nacen, se hacen". En Ponce Ceballos y Alcantar Enríquez (Coords.) La Formación de Profesores. Propuestas y respuestas. (U. A. California, Ed.) México: Juan Pablos Editor SA.

* Pérez Rivera, G. (1987). La formación docente en la perspectiva del Centro de Didáctica y su proyección en el Centro de Investigaciones y Servicios Educativos (CISE) de la UNAM. Perfiles Educativos (38), 15.

- Rodríguez López, J. (2000). Bases y estrategias de formación docente del profesorado (Segunda Edición ed.). España: Hergé.

* Rosales Medrano, M., \& Rosales Badilla, D. (2013). Fundamentos conceptuales en la Formación Docente y para la profesionalidad docente. En M. Rosales Medrano, Teoría y práctica de la profesión docente (pp. 17-35). Universidad Pedagógica del Estado de Sinaloa.

- Sacristán, G. (s.f.). Profesionalización docente y cambio educativo. En Maestros. Buenos Aires: Miño y Dávila.

- Tenti Fanfani, E. (1995). Una carrera con obstáculos: la profesionalización docente. En Documento de trabajo. Universidad Pedagógica Nacional. México: UPN.

* Zarzar Charur, C. (1988). Formación de profesores universitarios. Análisis y evaluación de experiencias. México: SEP Nueva Imagen.

\section{Cómo citar este artículo}

* Torres Carrasco, Ruth (2018). Reflexiones sobre la formación docente de una profesora-administradora. Revista Digital Universitaria (RDU). Vol. 19, núm. 1 enerofebrero. DOI: http://doi.org/10.22201/codeic.16076079e.2018.v19n1.a3. 